Pacific Journal of Mathematics

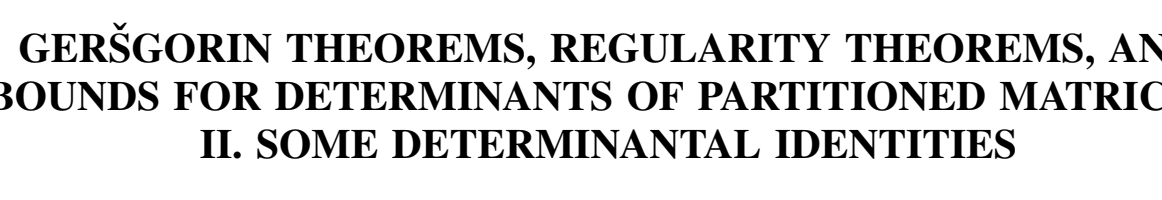




\title{
GERŠGORIN THEOREMS, REGULARITY THEOREMS, AND BOUNDS FOR DETERMINANTS OF PARTITIONED MATRICES \\ II \\ SOME DETERMINANTAL IDENTITIES
}

\author{
J. L. BRENNER
}

A square matrix $A=\left[a_{i j}\right]_{1}^{n}$ has dominant diagonal if $\forall i\left\{\left|a_{i i}\right|>R_{i}=\sum_{j \neq i}\left|a_{i j}\right|\right\}$. A more complicated type of dominance is the following. Suppose for each $i$, there is assigned a set $I(i)$ (subset of $\{1, \cdots, n\}$ ), $i \in I(i)$ : Define $B_{i j}$ as the $I(i) \times I(i)$ submatrix of $A$ that uses columns $I(i)$, and rows $\{I(i) \backslash i, j\}$, i.e., the set obtained from $I(i)$ by replacing the $i$ th row by the $j$ th row. Set $b_{i j}=\operatorname{det} B_{i j}$. Then $\left[b_{i j}\right]_{1}^{n}$ is a matrix, the elements of which are determinants of minor matrices of $A$. In an earlier paper, bounds for $\operatorname{det} A$ were derived in case $\left[b_{i j}\right]$ has dominant diagonal in the special case that $\{I(i)\}_{i}$ represents a partitioning of the indices into disjoint subsets.

In this article the general case is treated; $I(i)$ can be any subset of $\{1, \cdots, n\}$ that contains $i$. An identity is derived connecting $\operatorname{det}\left[b_{i j}\right]_{1}^{n}$ with $\operatorname{det} A$.

To establish the identity, a general multinomial identity is first derived, connecting determinants of certain submatrices of an $r \times 2 r$ matrix of indeterminates. This result, reminiscent of Sylvester's determinantal identity, is used to bound $\operatorname{det} A$.

1. Application of a characterization of the determinant function.

Lemma 1.01. Let $A=\left[a_{i j}\right]_{1}^{n}$ be a matrix of complex numbers [or indeterminates]; let a function $\phi: A \rightarrow C$ [or $\left.\phi: A \rightarrow C\left[a_{11}, \cdots, a_{n n}\right]\right]$ have the following properties for all $n \times n$ matrices $A$.

(1.02) [1.03] If any row [column] of $A$ is replaced by the sum of that row [column] and a multiple of another row [column], $\phi(A)$ is unaltered.

(1.04) If any row of $A$ is multiplied (throughout) by a constant $\alpha, \dot{\phi}(A)$ is multiplied $\alpha^{r}$.

Then $\phi(A)$ is a constant $c_{0}$ (independent of $a_{i j}$ ) multiplied by the rth power of $\operatorname{det} A$. 
Proof. The hypotheses $(1.02,1.03)$ guarantee that $\phi(A)$ is the same as $\phi(B)$, where $B$ is any matrix obtainable from $A$ by means of elementary transformations. It is known that $B=\operatorname{diag}[\operatorname{det} A, 1, \cdots, 1]$ can be so obtained; see for example [1]. Thus $\phi(A)$ is some function of $\operatorname{det} A$; the conclusion of lemma 1.01 follows on applying hypothesis 1.04 to the matrix $B$ : If $\phi(\alpha x)=\alpha^{r} \phi(x)$, then $\phi(x) \equiv c_{0} x^{r}$, since $\phi(x) / x^{r}$ is constant.

An application of this result was made in [2], to which the reader should refer. In slightly changed notation, this application is as follows.

LEMMA 1.05. Let $A=\left[a_{i j}\right]_{i=1, j=1}^{r 2 r}$ be an $r \times 2 r$ matrix of indeterminates, let $b_{i j}=\operatorname{det} A\left(\begin{array}{l}1 \cdots r \\ 1 \cdots i-1, i+1, \cdots, r, j\end{array}\right)$ be the determinant of the $r \times r$ submatrix of $A$ that uses columns $\{1, \cdots, r\} \backslash i, j$. This is the almost-principal submatrix of $A$ in which the ith column $i$ seplaced by the jth column. (For $j=i$, this is $A\left(\begin{array}{l}1 \cdots r \\ 1 \cdots r\end{array}\right)$. For $1 \leqq j \neq i \leqq r$, this submatrix has determinant 0 .)

Then

$$
X=\operatorname{det}\left[b_{i j}\right]_{i=1, j=r+1}^{\stackrel{r}{2} r}=G_{1}^{r-1} \operatorname{det}\left[a_{i j}\right]_{i=1, j=r+1}^{r}, \underset{2 r}{,},
$$

where

$$
G_{1}=\operatorname{det}\left[a_{i j}\right]_{11}^{r r}
$$

Note that in 1.06, the column indices are $r+1, \cdots, 2 r$.

To prove this Lemma, it is only necessary to observe that it is a multinomial identity, and that the hypotheses of Lemma 1.01 concerning the function $X$ are satisfied.

$1^{\circ}$ if $X$ is regarded as a function of $\left\{a_{i j}, 1 \leqq i, j \leqq r\right\}$;

$2^{\circ}$ if $X$ is regarded as a function of $\left\{a_{i j}, 1 \leqq i \leqq r, r<j \leqq 2 r\right\}$.

COROLlaRY 1.07. With the same hypothesis, the conclusion

$$
Y=\operatorname{det}\left[b_{i j}\right]_{i=1, j \in S}^{r}=G_{1}^{r-1} \operatorname{det}\left[a_{i j}\right]_{i=1, j \in S}^{r}
$$

is valid, where $S$ is any set of $r$ distinct positive integers not exceeding $2 r$.

Proof. Since 1.06 is a multinomial identity, the $r^{2}$ indeterminates $a_{i j}(j>r)$ on the right can simply be replaced by the $r^{2}$ indeteminates $a_{i j}(j \in S)$. But this replacement changes not only the range of $j$ in the set variables $\left\{a_{i j}\right\}$, but also the range of $j$ in the set $\left\{b_{i j}\right\}$, as the definition of $b_{i j}$ shows. 
Lemma 1.09. Suppose

$$
I(1)=\{1\}, I(2)=\{1,2\}, \cdots, I(r)=\{1,2, \cdots, r\} .
$$

Let $B=\left\{b_{i j}\right]_{i=1, j=1}^{r} \underset{2 r}{2 r}$ be defined as in 1.05. Then

$$
\begin{aligned}
& \operatorname{det} B\left(\begin{array}{c}
1, \cdots, r \\
r+1, \cdots, 2 r
\end{array}\right) \\
= & a_{11} \operatorname{det} A\left(\begin{array}{c}
12 \\
12
\end{array}\right) \operatorname{det} A\left(\begin{array}{l}
123 \\
123
\end{array}\right) \cdots \operatorname{det} A\left(\begin{array}{c}
12 \cdots r-1 \\
12 \cdots r-1
\end{array}\right) \\
& \times \operatorname{det} A\left(\begin{array}{c}
1,2, \cdots, r \\
r+1, \cdots, 2 r
\end{array}\right) .
\end{aligned}
$$

REMARK. This is again a multinomial identity in the $2 r^{2}$ indeterminates $a_{i j}$. Therefore 1.09 has the Corollary

(1.11) $\operatorname{det} B\left(\begin{array}{ccc}1 & \cdots & r \\ j_{1} & \cdots & j_{r}\end{array}\right)=a_{11} \operatorname{det} A\left(\begin{array}{l}12 \\ 12\end{array}\right) \operatorname{det} A\left(\begin{array}{l}123 \\ 123\end{array}\right) \cdots \operatorname{det} A\left(\begin{array}{ccc}1 & \cdots & \cdots \\ j_{1} & \cdots & j_{r}\end{array}\right)$ in view of the definition of $b_{i j}$.

Proof of Lemma 1.09. To show that $a_{11}$ is a factor in (1.10), as shown, $a_{21}$ times the first row is added to the second row. The second row becomes

$$
a_{11} a_{2, r+1}, a_{11} a_{2, r+2}, \cdots, a_{11} a_{2, r+j}, \cdots
$$

which obviously has $a_{11}$ as a factor.

It is a little more complicated to show $\operatorname{det}\left(\begin{array}{c}a_{11} a_{12} \\ a_{21} a_{22}\end{array}\right)$ is also a factor, as is asserted in relation (1.10). The trick is to add to the third row $-\operatorname{det}\left(\begin{array}{l}a_{21} a_{22} \\ a_{31} a_{32}\end{array}\right)$ times the first row as well as $a_{11}^{-1} \operatorname{det}\left(\begin{array}{l}a_{11} a_{12} \\ a_{31} a_{32}\end{array}\right)$ times the second row (1.13). The new third row is

$$
\operatorname{det}\left(\begin{array}{c}
a_{11} a_{12} \\
a_{21} a_{22}
\end{array}\right)\left[a_{3, r+1}, a_{3, r+2}, \cdots, a_{3, r+j}, \cdots\right],
$$

i.e., every element of that row has the common prefactor indicated.

The formal proof of (1.10) is inductive, as follows. As an induction hypothesis, assume that the left member of (1.10) can be written in the form

$$
a_{11} \operatorname{det} A\left(\begin{array}{l}
12 \\
12
\end{array}\right) \cdots \operatorname{det} A\left(\begin{array}{c}
12 \cdots k-1 \\
12 \cdots k-1
\end{array}\right) \operatorname{det} C_{k},
$$

where $C_{k}$ is the $r \times r$ matrix, the $j$ th column of which is 


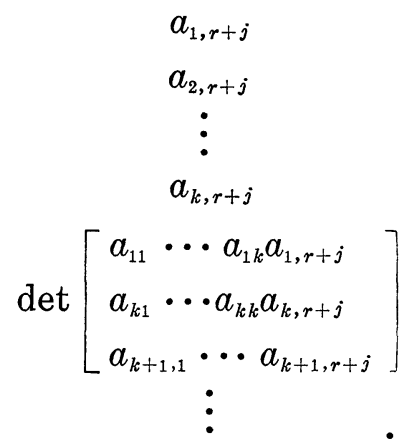

This has already been established for $k=1,2$. The inductive assertion is: the factor $\operatorname{det} A\left(\begin{array}{c}12 \cdots k \\ 12 \ldots k\end{array}\right)$ splits off from $\operatorname{det} C_{k}$. To prove this, subtract from the $k+1$ st row of the matrix $C_{k}$ appropriate multiples of the preceding rows. The multiple of $a_{i, r+j}$ needed is precisely the cofactor of $a_{i, r+j}$ in $C_{k}$ itself.

This completes inductive proof. To establish (1.10) in its entirety, a final visual check is needed of the circumstance that for $k=r$, the matrix $C_{r}$ is indeded the matrix $A\left(\begin{array}{c}1 \cdots r \\ r+1 \cdots 2 r\end{array}\right)$. See (1.05).

\section{Some special factorizations.}

THeORem 2.01. Let $A=\left[a_{i j}\right]$ be a matrix with $r$ rows: $i=1(1) r$, and $2 r$ columns: $j=1(1) r<j_{1}<\cdots<j_{r}$. Suppose, for $i=1,2, \cdots, r-1$, $I(i)=\{1,2, \cdots, r-1\} ; I^{\prime}(r)=\{1,2, \cdots, r\}$. For $j=j_{1}, j_{2}, \cdots, j_{r}$ set $B_{i j}=A\left(\begin{array}{l}I(i) \\ I(i) \backslash i, j\end{array}\right), i=1(1) r$.

Denote $\operatorname{det} B_{i j}$ by $b_{i j} ; B=\left[b_{i j}\right]$. Then

$$
\operatorname{det} B= \pm C^{r-1} \operatorname{det} A\left(\begin{array}{c}
1,2, \cdots, r \\
j_{1} j_{2} \cdots j_{r}
\end{array}\right) ; C=\operatorname{det} A\left(\begin{array}{l}
I(1) \\
I(1)
\end{array}\right) \text {. }
$$

Proof. Consider the last row of $B$. The element $b_{r j}$ in column " $j$ " of this row is the determinant of the $r \times r$ matrix $B_{r j}$. If this determinant is expanded by minors of the elements $a_{r j}, a_{r 1}, a_{r 2}, \cdots a_{r, r-1}$ of the last row of $B_{r j}$, the result is

$$
b_{r j}= \pm a_{r j} C \pm a_{r 1} b_{1 j} \pm a_{r 2} b_{2 j} \pm \cdots \pm a_{r, r-1} b_{r-1, j} \cdot
$$

Relation (2.03) shows that $\operatorname{det} B$ is not altered if every element $b_{r j}$ of the last row of $B$ is replaced by $\pm a_{r j} C$. (This replacement would merely omit from the last row of $B$ a linear combination of the preceding rows.)

At this point it is clear that $C$ is a factor of $\operatorname{det} B$, and that the other factor has the same first $r-1$ rows does $B$, and has last 
row $a_{r j}$. The conclusion of the theorem now follows by expanding $\operatorname{det} B$ by its last row and applying Corollary 1.07. See Lemmas 4.3, 4.4 of [2].

Corollary 2.04. Suppose

$$
I(i)=\{1,2, \cdots, r-k\} \text { for } i=1,2, \cdots, r-k ;
$$

and $I(i)=\{1,2, \cdots, r-k, i\}$ for $i=r-k+1, \cdots, r$. Then (2.02) holds; where $C$ now means $\operatorname{det} A\left(\begin{array}{l}1,2, \cdots, r-k \\ 1,2, \cdots, r-k\end{array}\right)$.

2.05. Another special case is the case $I(1)=\{1,2\}, I(2)=\{2,3\}$, $I(3)=\{3,1\}$. The formula

$$
\operatorname{det} B=G \operatorname{det} A, \quad G=\operatorname{det}\left[\begin{array}{ccc}
a_{11} & -a_{12} & 0 \\
0 & a_{22} & -a_{23} \\
-a_{31} & 0 & a_{23}
\end{array}\right]
$$

can be verified by appropriate devices. A generalization of (2.06) is the formula

$$
\operatorname{det} B\left(\begin{array}{lll}
1 & 2 & 3 \\
j_{1} & j_{2} & j_{2}
\end{array}\right)=G \cdot \operatorname{det} A\left(\begin{array}{lll}
1 & 2 & 3 \\
j_{1} & j_{2} & j_{3}
\end{array}\right),
$$

valid for any $3 \times 6$ matrix $A$, with $I(i)$ defined as above. Among several valid proofs of this formula, the following is presented. It proves (2.07) as a special case of a still more general result.

Theorem 2.08. Let $A=\left[a_{i j}\right]$ be an $r \times 2 r$ matrix, $i=1(1) r, j=$ 1(1)2r. Let $B$ be the $r \times r$ matrix with $(i, j)$ element $b_{i j}=\operatorname{det} B_{i j}$, where $B_{i j}=A\left(\begin{array}{cc}i & i+1 \\ j & i+1\end{array}\right), i=1(1) r-1, B_{r j}=A\left(\begin{array}{c}r 1 \\ j 1\end{array}\right) ; j=r+1(1) 2 r$. Then the relation

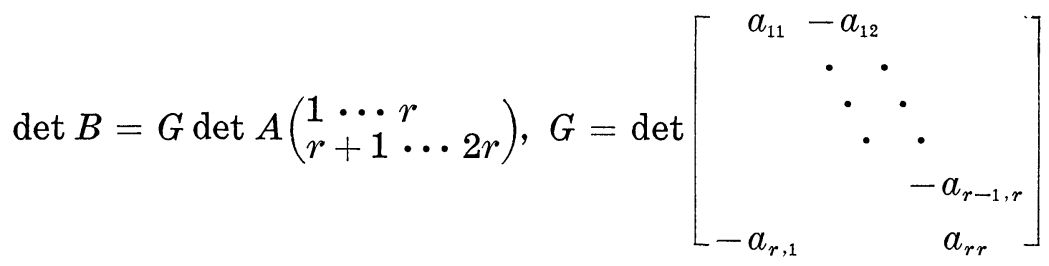

holds; $G$ is a bidiagonal matrix with $2 r$ nonzero elements.

REMARK. This is the case $I(1)=\{1,2\}, I(2)=\{2,3\}, \cdots, I(r)=$ $\{r, 1\}$.

Proof. Subtract a multiple of the first row of $B$ from the second, then a multiple of the second from the third, ..., a multiple of the 
$r-1 s t$ from the last. The resulting matrix has the same determinant as $B$, and the multiples mentioned can be chosen so that this resulting matrix is, row by row,

$$
\begin{array}{rc}
a_{22}\left[a_{1 j}\right]-a_{12}\left[a_{2 j}\right] & \cdots 1 \\
\left(a_{22} a_{33} / a_{12}\right)\left[a_{1 j}\right]-a_{23}\left[a_{3 j}\right] & \cdots 2 \\
\left(a_{22} a_{33} a_{44} / a_{12} a_{23}\right)\left[a_{1 j}\right]-a_{34}\left[a_{4 j}\right] & \cdots 3 \\
\vdots & \vdots \\
\left(a_{11} a_{22} \cdots a_{r r} / a_{12} \cdots a_{r-1 r}-a_{r 1}\right)\left[a_{1 j}\right] & \cdots r .
\end{array}
$$

Now subtract a multiple of the new last row from each of the preceding rows; the first $r-1$ rows of the new matrix are $-a_{12}\left[a_{2 j}\right]$, $-a_{23}\left[a_{3 j}\right], \cdots$. This matrix obviously has determinant (2.09). $\|$

3. General factorization of $\operatorname{det} B$. The function $i \mapsto I(i)$ induces a (weak) separation of the indices $\{1, \cdots, n\}$ into agglomerated mutually exclusive sets $S(k)$, as follows.

Definition 3.01. Let $i \mapsto I(i)$ be a function from the integers $\{1, \cdots, n\}$ to sets of these same integers, with the further property $i \in I(i)$ for all $i$. In the usual way, the sets $I(i)$ are now agglomerated into the smallest possible (minimal) mutually exclusive sets $S(k)$ so that:

Every $I(i)$ is in one or another of the sets $S(k)$. Then $S(k)$ are the mutually separated sets defined by the function I. For example, the function

$$
\begin{aligned}
& 1 \longmapsto\{1\}, 2 \longmapsto\{1,2\}, 3 \longmapsto\{1,2,3\}, 4 \longmapsto\{4,5\}, 5 \longmapsto\{5,6\}, \\
& 6 \longmapsto\{6,7\}, 7 \longmapsto\{7\}
\end{aligned}
$$

defines a separation of the indices $\{1,2,3,4,5,6,7\}$ into the mutually exclusive sets $S(1)=\{1,2,3\}, S(2)=\{4,5,6,7\}$.

Parallel to the separation of Definition 3.01, there is a factorization of $\operatorname{det} B$ into a product of factors, one for each set $S(k)$. The $k$ th factor is the determinant of a matrix; in general the elements of this matrix are again determinants of matrices: the elements of these matrices are elements $a_{i j}$ of the matrix $A$, where $i, j \in S(k)$. The point is that the polynomial function $\operatorname{det} B$ of the elements of $A$ factors into the product of multinomial factors; the $k$ th factor is a polynomial in the indeterminates $a_{i j}$, where $i, j$ belong only to the $k$ th set $S(k)$ of indices. Besides these factors, $\operatorname{det} A$ also appears as a factor.

It there are two or more sets $S(k)$ in the separation, then $\operatorname{det} A$, but not $(\operatorname{det} A)^{2}$, is thus a factor of $\operatorname{det} B$. Even when the entire set $\{1,2, \cdots, n\}$ of indices are connected through the sets $I$ (there is but a single set $S$ ), the factor $\operatorname{det} A$ appears only to first power "in 
general." The exact meaning of "in general" is explained below.

The above remarks are summarized in the following theorem. Its proof, together with a more detailed atatement, unfold in $\S 4$.

THEOREM 3.02. Let $A=\left[a_{i j}\right]$ be an $n \times n$ matrix of indeterminates; for $i=1(1) n$ let $I(i)$ be a subset of the first $n$ integers with $i \in I(i)$. Denote by $B_{i j}$ the minor $A\left(\begin{array}{l}I(i) \\ I(i) \backslash i, j\end{array}\right)$ on rows $I(i)$; and on columns $I(i)$, but with index $i$ replaced by $j$. Set $b_{i j}=\operatorname{det} B_{i j} ; B=\left[b_{i j}\right]$. Thus $B$ is an $n \times n$ matrix. Let the function $I(i)$ induce a separation of the indices $\{1, \cdots, n\}$ into $s \geqq 1$ mutually exclusive sets $S_{1}, S_{2}, \cdots, S_{s}$. Then $\operatorname{det} B$, which is obviously a polynomial function of the $n^{2}$ indeterminates $a_{i j}$ with integer coefficients, can be factored in the form

$$
\operatorname{det} B=G \operatorname{det} A \text {, }
$$

where $G=M_{1} M_{2} \cdots M_{s}$, and where each $M_{k}$ is a multinomial in those indeterminates $a_{i j}$ for which both indices $i, j$ belong to the set $S_{k}$. In particular, $\operatorname{det} A$ is always a factor of $\operatorname{det} B$.

The details of the proof depend on the following lemma.

LEMmA 3.03. Let $A=\left[a_{i j}\right]$ be an $r \times 2 r$ matrix of indeterminates, $i=1(1) r, j=1(1) 2 r$. For each $i$, let $I(i)$ be a subset of the first $r$ integers. Let $B_{i j}, b_{i j}$ be defined formally as in Theorem 3.02. $B_{1}$ is the $r \times r$ matrix $\left[b_{i j}\right], 1 \leqq i \leqq r<j \leqq 2 r . A_{1}$ is the $r \times r$ matrix $\left[a_{i j}\right]_{1 \leqq i \leqq r<j \leqq 2 r}$. (Note the range for $j$.)

Then the polynomial identity

$$
\operatorname{det} B_{1}=F \cdot \operatorname{det} A_{1}
$$

holds, where $F$ is a multinomial with integer coefficients in the indeterminates $\left\{a_{i j}, 1 \leqq i, j \leqq r\right\}$.

REMARK 3.05. This lemma is more general than any of previous ones, since the sets $I(i)$ are more general.

Corollary 3.06. Det $A_{1}$ is, but $\left(\operatorname{det} A_{1}\right)^{2}$ is not a factor of $\operatorname{det} B_{1}$.

Proof. The variables that figure in $F$ are disjoint from those in $B_{1}$.

REMARK 3.07. This is the meaning of the phrase "in general" above.

Corollary 3.08. Let $A_{1}, B_{1}$ redefined conformally. That is, 
without changing the sets $I(i)$, let the range for $j$ in the definitions of $A_{1}, B_{1}$ be replaced by any range of $r$ distinct integers, including some or all of the first $r$ integers. Then (3.04) still holds.

Proof. If some of the indices $j$ in the polynomial $\operatorname{det} A_{1}$ are changed, the definition of $b_{i j}$ shows that a conformal change is concurrently made in the polynomial $\operatorname{det} B_{1}$. In other words, the change amounts solely to a change of the names of the variables in (3.04). But (3.04) is a polynomial identity.

Under the change $a_{i, j} \rightarrow a_{i, j-r}, b_{i, j} \rightarrow b_{i, j-r}$ in (3.04), the factor $\operatorname{det} A_{1}$ could appear as a factor in $F$ for suitable choice of $I(i)$. For example, if $I(i) \equiv\{1,2, \cdots, r\}$, and if $j$ runs through the range $1 \leqq$ $j \leqq r$, then (3.04) becomes $\operatorname{det} B_{1}=\left(\operatorname{det} A_{1}\right)^{r}$.

Proof of Lemma 3.03. To avoid difficulties with an algebraic sign, the columns of $B_{i j} \equiv A\left(\begin{array}{l}I(i) \\ (I(i) \backslash i, j\end{array}\right)$ are to be thought of as written in a definite order: the $j$ th column $a_{i j}$ first, followed by the other columns in natural order. For example, if $I(1)=\{1,2,3\}$ then $B_{1 j}$ is the matrix

$$
\left[\begin{array}{lll}
a_{1 j} & a_{12} & a_{13} \\
a_{2 j} & a_{22} & a_{23} \\
a_{3 j} & a_{22} & a_{33}
\end{array}\right] .
$$

Without this convention, the formula to be obtained for $F$ would be determined only up to sign.

It will be instructive to carry through the proof in a special case, since a rather simple special case already embodies all the points of difficulty and interest. The case $I(1)=\{1,2\}, I(2)=\{1,2,3\}, I(3)=$ $\{1,2,3\}$ will serve as an illustration. The matrix $B_{1}$ has as $j$ th column $B_{1 j}$, where

$$
B_{1 j}=\left[\begin{array}{l}
\operatorname{det}\left[\begin{array}{ll}
a_{1 j} & a_{12} \\
a_{2 j} & a_{22}
\end{array}\right] \\
\operatorname{det}\left[\begin{array}{lll}
a_{1 j} & a_{11} & a_{13} \\
a_{2 j} & a_{21} & a_{23} \\
a_{3 j} & a_{31} & a_{33}
\end{array}\right] \\
\operatorname{det}\left[\begin{array}{lll}
a_{1 j} & a_{11} & a_{12} \\
a_{2 j} & a_{21} & a_{22} \\
a_{3 j} & a_{31} & a_{32}
\end{array}\right]
\end{array}\right]_{j=4,5,6 .}
$$

The first step in the proof is to border the $3 \times 3$ matrix $B_{1}$ with 3 rows and columns as shown below. The enlarged matrix $B_{2}$ clearly has the same determinant as $B_{1}$, except for the factor $(-1)^{r}$. Only 
the subscripts are printed; thus $1 j$ is an abbreviation for $a_{1 j}$. The reader must also supply the symbol det throughout: [ ] is an abbreviation for $\operatorname{det}[$ ].

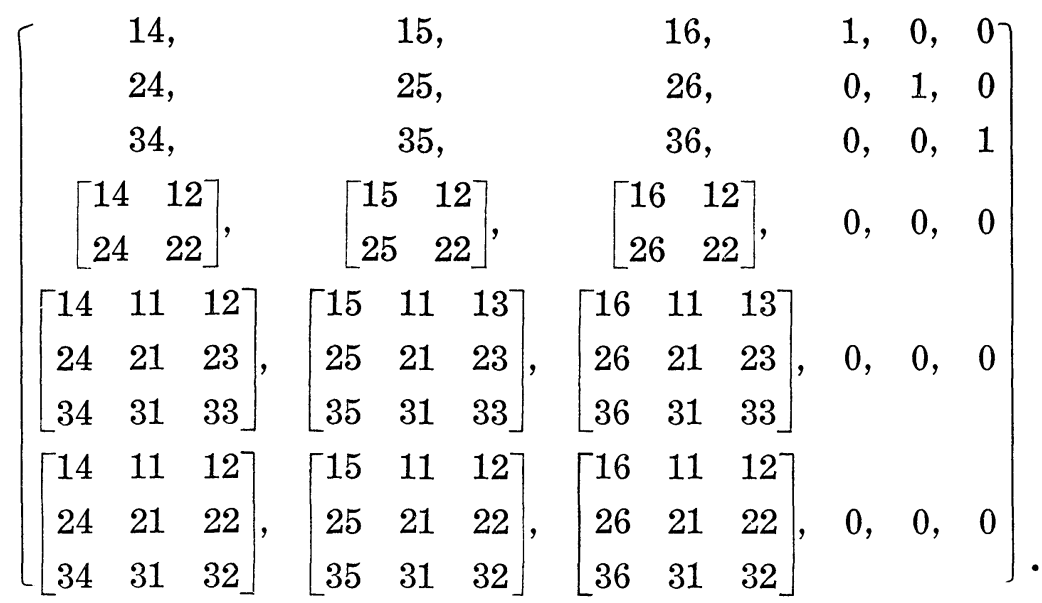

To show that the factor $\operatorname{det} A_{1}$ splits off from the determinant of this $6 \times 6$ matrix, it need only be noted that the matrix can be reduced to the form $\left[\begin{array}{cc}A_{1} & I \\ 0 & F_{1}\end{array}\right]$ by adding appropriate linear combinations of the first three rows to each of the last three. This argument is an alternative to a general argument of Loewy [3], who proved by another method that if $\operatorname{det} A_{1}=0$, then necessarily $\operatorname{det} B_{1}=0$. In the special case being expounded, $\operatorname{det} B_{2}=-\left(\operatorname{det} F_{1}\right)\left(\operatorname{det} A_{1}\right)$, where $F_{1}$ is the $3 \times 3$ matrix

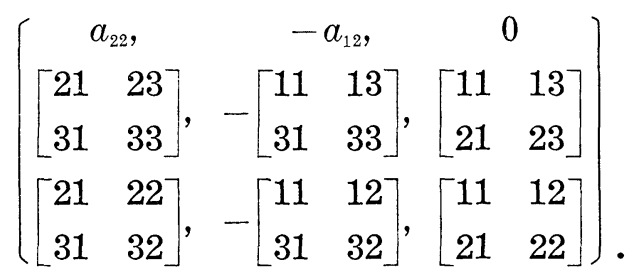

The argument given above has general applicability. Formula (3.04) is established. The multinomial $F$ is in fact the determinant of an $r \times r$ matrix. The $(k, l)$ element of this matrix is the negative of the cofactor of $a_{l \cdot r+l}$ in $b_{k, r+l}=\operatorname{det} A\left(\begin{array}{l}I(k) \\ I(k) \backslash k, r+l\end{array}\right)$, and is thus

$$
f_{k l}=-(-1)^{1+\operatorname{pos} l} \operatorname{det} A\left(\begin{array}{l}
I(k) \backslash l \\
I(k) \backslash k
\end{array}\right),
$$

where pos $l$ is the position of $l$ in the set $I(k)$. If $l \notin I(k)$, then $f_{k l}=0$, and conversely. For consistency, $f_{k k}$ must be defined as 1 when $I(k)=\{k\}$. 
CoRollaries.

$$
\operatorname{det} B_{1}=(-1)^{r}\left(\operatorname{det} F_{1}\right)\left(\operatorname{det} A_{1}\right)
$$

3.10 [3] If $\operatorname{det} A_{1}=0$, then $\operatorname{det} B_{1}=0$.

3.11. If $F_{1}$ is a triangular matrix, then

$$
\begin{gathered}
\operatorname{det} B_{1}=-(-1)^{r} \Pi\left(\operatorname{det} G_{1}^{(i)}\right) \cdot\left(\operatorname{det} A_{1}\right) \text {, where } \\
G_{1}^{(i)}=A\left(\begin{array}{l}
I(i) \backslash i \\
I(i) \backslash i
\end{array}\right) \cdot
\end{gathered}
$$

In particular, relation (1.10) follows; this proof differs from the first proof.

(3.14) In case $I(1)=\{1,2\}, I(2)=\{2,3\}, \cdots, I(i)=\{i, i+1\}, \cdots, I(n)=$ $\{n, 1\}$, then formula

(3.15) $\operatorname{det} B_{1}=G \cdot \operatorname{det} A_{1}$ holds, where $G=\operatorname{det}\left[\begin{array}{c}a_{11}-a_{12} \\ a_{22} \\ .\end{array}-a_{23}\right]$ is the determinant of the bidiagonal matrix shown. This proof is again different from the earlier proof of (2.09).

3.16. Note that the case $I(1)=\{1,2,3\}, I(2)=\{2,3,4\}, \cdots$ is considerably more complicated than the case (3.14); indeed while the first type of proof is more direct for the hypothesis (3.14), an attempt to generalize this proof to the case (3.16) is unrewarding.

\subsection{Relation (1.06) holds.}

The following proof of 1.06 is somewhat less direct than the original proof. The matrix $F_{1}$ is not triangular, so that the determinant $\operatorname{det} F_{1}$ does not factor for this simple reason. However $F_{1}$ is seen on inspection to be the $r-1 s t$ compound of the matrix $A\left(\begin{array}{l}I(1) \\ I(1)\end{array}\right)$; thus $\operatorname{det} F_{1}=\operatorname{det} A\left(\begin{array}{l}I(1 \\ I(1\end{array}\right)^{r-1}$. This proof requires a knowledge of the formula

(3.18) $\operatorname{det} C^{(t)}=(\operatorname{det} C)^{e}, e=\left(\begin{array}{c}r-1 \\ t-1\end{array}\right)$, where $C^{(t)}$ is the $t$ th compound of the $r \times r$ matrix $C$.

4. General factorization of $\operatorname{det} B$ (continued). In this section, Corollary 3.08 is applied to obtain a general formula for the determinant of the $n \times n$ matrix $B=\left[b_{i j}\right]$ defined in Theorem 3.02.

Since Theorem 3.02 holds for a matrix $A$ of indeterminates, it 
holds in particular for a matrix $A$ of complex numbers.

Proof of Theorem 3.02. The function $i \mapsto I(i)$ induces a separation of the indices $\{1,2, \cdots, n\}$ into $s \geqq 1$ mutually exclusive sets $S(k)$ such that every set $I(i)$ is in exactly one of the sets $S(k)$, and the sets $S(k)$ cannot be further decomposed without destroying these properties.

In following the details of the proof, the reader may prefer to think of the indices of the sets $S(1), S(2), \ldots$ as occuring in natural order.

To continue the proof, the rows of $B$ are partitioned into (mutually exclusive) sets $S(1), S(2), \cdots$ and $\operatorname{det} B$ is expanded according to the generalized Laplace expansion on these rows. Corollary 3.08 asserts that the determinants of all the $S(1) \times S(1)$ minor matrices on the set of rows with indices in $S(1)$ have a common factor $M_{1}$. The corollary asserts further that this common factor is a multinomial in the particular variables $a_{i j}(i, j \in S(1))$. Similarly for $S(2), \cdots$. Thus $M_{1} M_{2} \cdots M_{s}$ is a factor of $\operatorname{det} B$.

Besides the factor common to the determinants of all the $S(1) \times$ $S(1)$ matrices, there is a factor, see (3.04), peculiar to the particular minor matrix. This peculiar factor is just what is needed, in the Laplace expansion of $\operatorname{det} B$, to produce $\operatorname{det} A$. The proof of Theorem 3.02 is complete.

Let $A$ be a matrix of indeterminates. If there is more than one set $S(k)$, then $\operatorname{det} A$ is, but $(\operatorname{det} A)^{2}$ is not, a factor of $\operatorname{det} B$.

5. Applications. Theorem 3.02 can be used to obtain bounds for $\operatorname{det} A$ in case the matrix $B$ has dominant diagonal. The details and results are similar to those of [2]. These results have one remarkable feature: This is the first occasion on which such bounds have been obtained for a "partitioning" of a matrix, in which the sets of rows in the "partitioning" overlap one another.

The results of this paper will be needed in any attempt to obtain minimal Geršgorin sets related to the Hoffman-Brenner theorem. If it can be accomplished, this will be an interesting generalization of the results of [5].

\section{REFERENCES}

1. J. L. Brenner, Applications of the Dieudonné Determinant, Linear Algebra and its Applications 1 (1968), 511-536.

2. - Geršgorin theorems, regularity theorems, and bounds for determinants of partitioned matrices, SIAM J. Appl. Math., 18 (1970), 443-450.

3. F. R. Gantmacher, Applications of the theory of matrices, N. Y.: Interscience (Wiley) 1959. 
4. R. Loewy, Gerschgorin-type theorems for partitioned matrices, SIAM J. Num. Anal., 7 (1970), 125-128.

5. R. S. Varga, Minimal Gerschgorin sets, Pacific J. Math., 15 (1965), 719-729.

Received September 10, 1970, and in revised form April 13, 1971. Supported by NSF-GP9483.

UNIVERSITY OF ARIZONA

AND

UNIVERSITY OF VICTORIA 


\section{PACIFIC JOURNAL OF MATHEMATICS}

\section{EDITORS}

H. SAMELSON

Stanford University

Stanford, California 94305

C. R. HobBY

University of Washington

Seattle, Washington 98105
J. DugundjI

Department of Mathematics

University of Southern California

Los Angeles, California 90007

RICHARD ARENS

University of California

Los Angeles, California 90024

\section{ASSOCIATE EDITORS}

E. F. BECKENBACH

B. H. NeUMaNN

F. WOLF

K. YoshidA

\section{SUPPORTING INSTITUTIONS}

UNIVERSITY OF BRITISH COLUMBIA

CALIFORNIA INSTITUTE OF TECHNOLOGY

UNIVERSITY OF CALIFORNIA

MONTANA STATE UNIVERSITY

UNIVERSITY OF NEVADA

NEW MEXICO STATE UNIVERSITY

OREGON STATE UNIVERSITY

UNIVERSITY OF OREGON

OSAKA UNIVERSITY
UNIVERSITY OF SOUTHERN CALIFORNIA STANFORD UNIVERSITY

UNIVERSITY OF TOKYO

UNIVERSITY OF UTAH

WASHINGTON STATE UNIVERSITY UNIVERSITY OF WASHINGTON

$\stackrel{*}{*} \stackrel{*}{*} \stackrel{*}{*}$ AMERICAN MATHEMATICAL SOCIETY
NAVAL WEAPONS CENTER 


\section{Pacific Journal of Mathematics}

Vol. 39 , No. 1

May, 1971

Charles A. Akemann, A Gelfand representation theory for $C^{*}$-algebras ....

Sorrell Berman, Spectral theory for a first-order symmetric system of

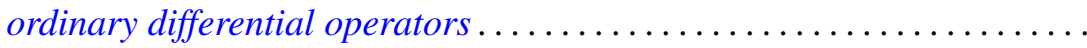

Robert L. Bernhardt, III, On splitting in hereditary torsion theories ........

J. L. Brenner, Geršgorin theorems, regularity theorems, and bounds for determinants of partitioned matrices. II. Some determinantal identities ..........................................

Robert Morgan Brooks, On representing $F^{*}$-algebras .............. 51

Lawrence Gerald Brown, Extensions of topological groups........... 71

Arnold Barry Calica, Reversible homeomorphisms of the real line ........ 79

J. T. Chambers and Shinnosuke Oharu, Semi-groups of local Lipschitzians in

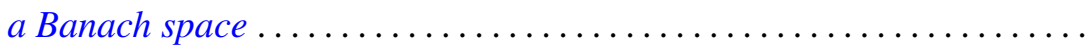

Thomas J. Cheatham, Finite dimensional torsion free rings .............

Byron C. Drachman and David Paul Kraines, A duality between

transpotence elements and Massey products ...................

Richard D. Duncan, Integral representation of excessive functions of a

Markov process ......................................

George A. Elliott, An extension of some results of Takesaki in the reduction

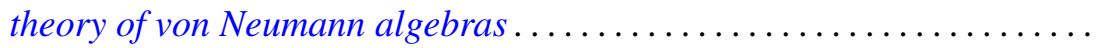

Peter C. Fishburn and Joel Spencer, Directed graphs as unions of partial

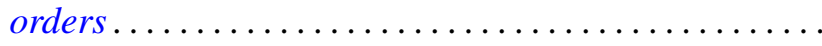

Howard Edwin Gorman, Zero divisors in differential rings ...

Maurice Heins, A note on the Löwner differential equations...

Louis Melvin Herman, Semi-orthogonality in Rickart rings. .

David Jacobson and Kenneth S. Williams, On the solution of linear G.C.D.

equations

Michael Joseph Kallaher, On rank 3 projective planes ... . .

Donald Paul Minassian, On solvable $O^{*}$-groups ...........

Nils Øvrelid, Generators of the maximal ideals of $A(\bar{D})$

Mohan S. Putcha and Julian Weissglass, A semilattice decomposition into

semigroups having at most one idempotent ............

Robert Raphael, Rings of quotients and $\pi$-regularity ....

J. A. Siddiqi, Infinite matrices summing every almost periodic sequence. .

Raymond Earl Smithson, Uniform convergence for multifunctions ...

Thomas Paul Whaley, Mulitplicity type and congruence relations in

universal algebras...

Roger Allen Wiegand, Globalization theorems for locally finitely generated modules... 